\title{
Development of Full Automatic Assembly System for Bulb Lamp Based on CPAC
}

\author{
Li Gen ${ }^{1, a}$, Wu Ziyue ${ }^{1, b, *}$, Li Hang ${ }^{1, c}$, Zhang Shuaiyang,,d, Luo Qiuhui ${ }^{1, e}$ \\ ${ }^{1}$ School of Mechatronics Engineering, He’nan University of Science \& Technology, He’nan \\ Luoyang, China \\ a lyligen520@163.com, b wuziyuetutu@126.com, c 2390064710@qq.com,

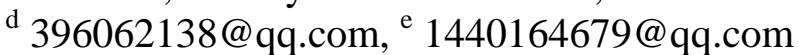 \\ *corresponding author
}

Keywords: LED; Full Automatic Assembly; Oto-studio; CPAC; PLC

\begin{abstract}
With the extensive application of LED bulb in life, the traditional artificial assembly production has been unable to meet the current living needs. According to the structural characteristics of LED bulb, the assembly line is designed and the three-dimensional solid modeling and simulation is completed. With the configuration software Oto-studio as the development platform, based on the CPAC servo experiment platform, the system development and human-computer interaction interface are designed to achieve LED bulb assembly automation operation. The experimental results show that: the system is stable and can realize the effective control and motion simulation of each action of the assembly point on the LED bulb assembly line, which has high practical value and wide market prospect.
\end{abstract}

\section{Introduction}

LED (Light Emitting Diode) Bulb lamp is a new type of lamp which can replace the traditional incandescent lamp. It has many advantages, such as energy saving, environmental protection, long life and small size. It is widely used in people's daily life [1].At present, most of the LED bulbs are assembly produced artificially in domestic enterprises, in order to solve the problem of low efficiency and unstable quality in LED bulb manual production, a set of LED bulb automatic assembly operation control system is worth to developing to improve production efficiency, ensure quality and reduce the economic cost. So far, the research of the whole LED bulb production line is still relatively little in our country.

The system are based on CPAC platform as the hardware development core, the software Oto-studio as the software platform the LED bulb assembly as the object, with the help of high-programmable automation controller, combined with modular design ideas, developing an open and easy to be transplanted LED bulb automated assembly system. The finished system should be able to achieve the effective control on the assembly point of the various operations and the state of monitoring and diagnosis in the LED bulb assembly line.

\section{Mechanical Design}

The LED bulb mainly includes the lamp module (including the driving power and inner sleeve), the lamp body, the light source board, the heat conduction plate and the fastening screw part, 
Wherein the outlet of the driving power source is welded to the light source board through the lamp body. According to the structural characteristics of the bulb, the top-down design sequence is used to determine the assembly process of lamp body loading, light source board installation, welding and fastening.

\subsection{Lamp Body Structural Design}

The station is designed to install the lamp body part. The structure mainly includes: fixture part, feeding part, installation part. The fixture is responsible for the flow among the line. Only when the previous process be completed, the fixture enter the next process or directly into the next work cycle; feeding part of the lamp body module forward delivery, supplement the material; the installation part composed the motor and cylinder, the cylinder is responsible for transferring the lamp body to the installation position, and then the motor switched between the lamp body and the lamp holder. As shown in Figure 1.

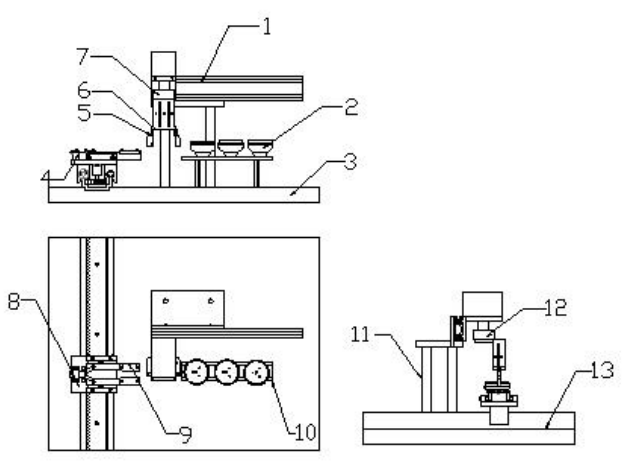

1. Pneumatic guide 2. Light body 3. Platform body

4. Clamp cylinder 5. Limit switch 6. Pneumatic manipulator 7.Stroke cylinder 8 Rack and Pinion 9 V-block

10. Feeding rack 11 Column 12. Stepper motor

13. Fixture guide

Figure 1 Mechanical structure of lamp body installation

\subsection{Design of Light Source Board Installation}

The light source board is the light emitting part of the LED lamp, which is to be installed, fastened and welded. As shown in Figure 2.

The installation of the light source board is to connect the light source board to the lamp body. The motion control of the station includes three motor switches and movement time control, cylinder switch and stroke control. Since the positioning of the light source board is high, the visual system is used to assist in the detection of the position of the positioning screw hole. The visual control is used for self-correction and achieving remote monitoring [2-3].

The fastening station is fastened to the thermally conductive plate of the lamp body by means of a screw mechanism. The body consists of four parts: feeder, screwdriver, screwdriver manipulator and controller. The screwdriver is mounted on the lock screw manipulator, connected to the feeder, and the electric screwdriver is controlled by the circuit connection via the controller. The rotary motion of the mechanical screw shaft is driven by the motor.

The welding station structure is a gantry uniaxial manipulator. The tin feed mechanism is mounted on the welding manipulator and the solder is sent to the welding head through the welding manipulator. The up and down movement of the welding manipulator of the station is driven by the 
motor. It is necessary to control the switching time, the movement time of the motor and the opening and closing of the preheating switch of the welding head.
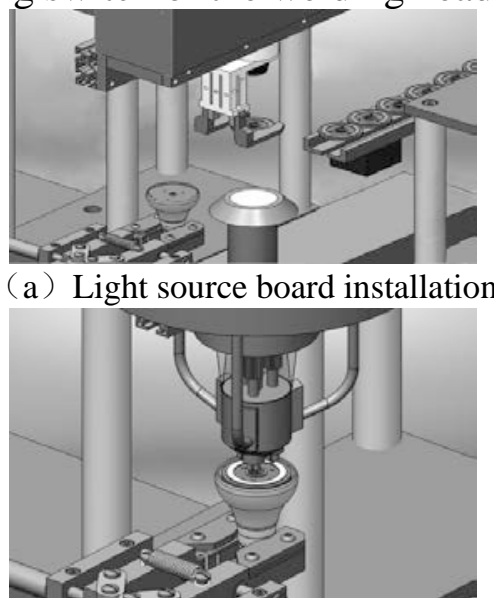

(b) Light plate fastening

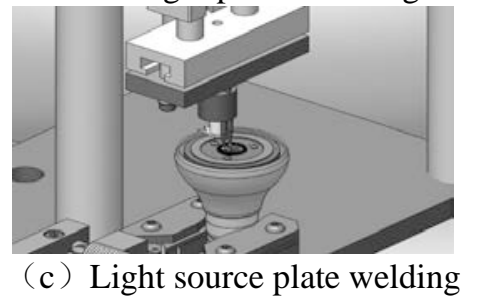

Figure 2 Mechanical structure of light source board

\subsection{Lamp Shade Structure Design}

The structure of the station is a gantry uniaxial mechanical straw, as shown in Figure 3. The movement of the station includes the upper and lower movement of the mechanical suction shaft, the suction movement of the straw. The upper and lower movement of the mechanical pipette shaft is driven by the motor, and the suction movement of the straw is driven by the cylinder. The motion control of the station requires control the motor's switching time, rotation time, cylinder opening and closing time and cylinder stroke. This process is similar to the lamp body loading process and is not described here.

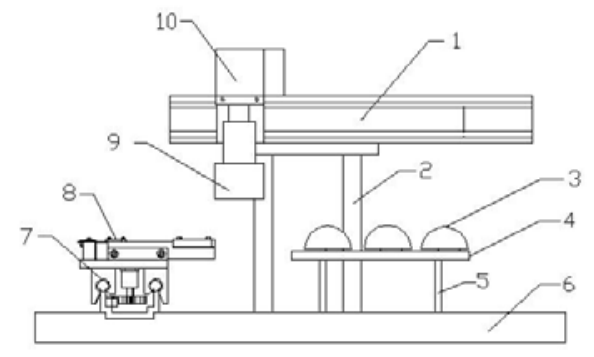

1. Pneumatic guide 2. pillar 3. lampshade 4. Feed rails

5. Feeding platform column 6. Fixed platform

7. Transmission guide

8.clamp body 9. straw 10. cylinder

Figure 3 Shade installation structure 


\section{System Hardware Design}

CPAC is the product of PC technology and motion control technology. It is combined with the standard X86 architecture and chipset for the system processor, using high-performance DSP and FPGA as a motion control coprocessor. It is highly integrated in a smaller controller, constitute a complete control system, can handle multi-axis operation, multi-signal processing, also achieve all the basic functions of ordinary PC [4]. The system architecture is shown in Figure 4.

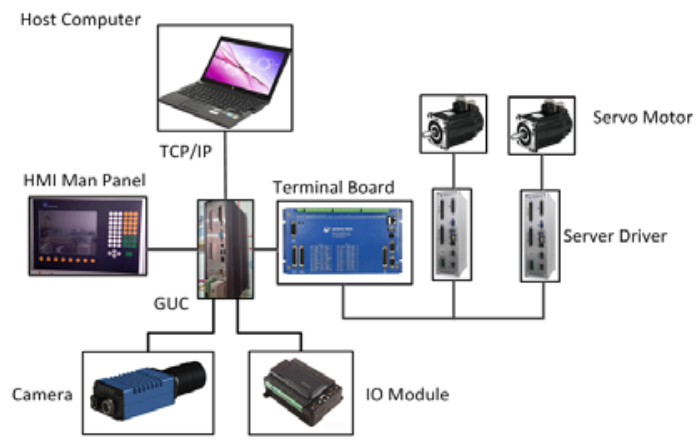

Figure 4 CPAC system architecture diagram

CPAC platform hardware consists of GUC series universal integrated controller, CNC man-machine display panel, servo motor system, power module and terminal block board and other components. In the host computer development software to write the program, compiled by the network cable can be directly downloaded to the program after the GUC controller, after the operation processing, the results back to the display panel.

\section{System Software Design}

The LED bulb assembly line system design is modular design, first of all the process of the process files written to the module, in order to order the logic of its integration. The whole system can be divided into motion control module, data processing module, state monitoring module and human-computer interface four modules [5-6].

The motion control module mainly carries on the plan to the movement of the motor and the cylinder, receives the feedback signal completes the movement control to the motor and the cylinder. Data processing module mainly includes sensor signal acquisition, A/D conversion and so on. The collected sensor signal is converted into digital quantity by software internal function, and then feedback back to the controller to form closed-loop control or directly as the result displayed on the man-machine interaction interface. The status monitoring module includes fault alarm unit and I/O unit. The I/O unit real-time monitors the entire assembly process. If there is an abnormality in the production process, the system will stop running and return the alarm information immediately. The man-machine interaction interface includes motion control interface, graphic interface and monitoring interface. In the human-computer interaction interface not only can control the production process, change the production parameters, but also real-time monitoring system operating conditions.

\section{System Testing and Simulation}

The test results can be displayed on the man-machine interface when system simulation running. The users can send the program, station parameters and other instructions to the system through the man-machine interface. The control system can feedback the parameters of the station movement status and fault alarm to the user through the man-machine interface to realize the control, 
adjustment and adjustment of the production process monitor, details as follows:

While the program start, the system automatically jumps to the main interface, mainly contains the coordinate information, alarm information, movement state information, manual operation area, key area and so on. Users can choose the motion control interface, graphics interface and IO interface, the interface can be switched between each other. The motion interface includes automatic operation and manual operation to display the motor real-time motion status. IO interface contains positive and negative limit signal, zero position capture signal and cylinder opening and closing signals. The graphic fit interface is used to simulate the operation of each station in the display assembly line. As shown in Figure 5.

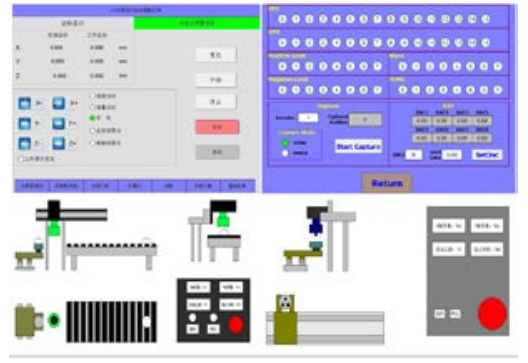

Figure 5 System main interface and motion fitting interface

In the case of the lamp body loading process, when the movement command is issued and the feeding is started, the main conveyance mechanism sends the lamp body to the designated position below the mechanical gripper along the fixture guide, the position of which is determined by the sensor, the stop mechanism is stopped, and the cylinder manipulator slow down, the distance set by the limit switch; in place after clamping the cylinder gently clamping the lamp body, the cylinder linkage mechanical grips up and up to move to the top of the fixture, the specific location by the proximity sensor identification; cylinder down To reach the designated location, the motor drive the handle and the lamp body to reverse the installation, the installation is complete, the robot back to the original position. The operational logic flow chart is shown in Figure 6 [7-9].

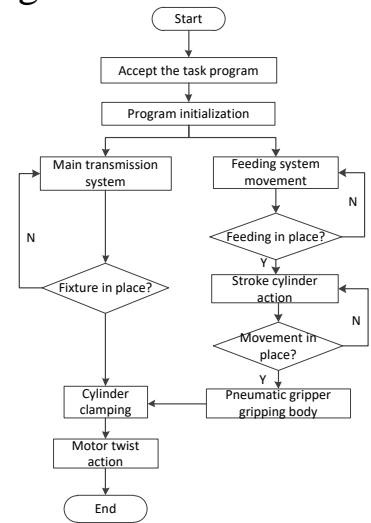

Figure 6 Logic flow chart of Lamp body loading operation

\section{Conclusion}

According to the structural characteristics of LED bulb, design an LED bulb automatic assembly system. The assembly process flow is determined according to the production object, and the assembly of the mechanical structure is designed. Based on the CPAC open test platform, the design and simulation of the whole assembly system was completed to realize the automatic operation of the assembly of the LED bulb, and the human-computer interaction interface was developed to facilitate the production management and remote operation. After testing, the system achieved the 
expected function, stable operation, good human-computer interaction. The system can be applied to the LED bulb structure similar to the other electronic components of the production process, a high practical value and market prospects, but also for the improvement of similar products to provide a reference.

\section{References}

[1] Tan Shuzhan, Chen Lina, Wang Dexi etc. The present development of LED lighting industry in China[J]. World Plastics.2013,31(3):41-43.

[2] Chu Letian,Zhu Lanjuan. Automatic assembly line of LED lamps based on machine vision[J].Computer CD Software and Applications, 2014,(23):127-129.

[3] Hao Yongpeng, Yu Daguo, Li Shaomin etc. Linearity Detecting System for Deep Holes Based on the Light Vision Technology[J]. Modular Machine Tool \& Automatic Manufacturing Technique. 2016,(2):51-53,59.

[4] Xu Yang. Development of Six Degrees of Freedom Open Robot Control System Based on CPAC[D]. Guangdong University of Technology,2013.

[5] Liu Lu, Chang Xiaoling. The PLC Control System Design of the AS Stacker Crane Based on the Industrial Control Networks[J]. Modular Machine Tool \& Automatic Manufacturing Technique.2015,(1):93-96.

[6] Miao Zeyu, Kong Fanyu, Li aiqin etc. The Design of Control System of Multi-station Brushing Machine Tool Based on PLC[J]. Modular Machine Tool \& Automatic Manufacturing Technique, 2016,(9):40-42,46.

[7] Soft Technology (Shenzhen) Co., Ltd. Otostudio Motion Control Library Programming Manual[Z].2010.

[8] Solid Technology (Shenzhen) Co., Ltd. Otostudio visual interface development manual [Z] .2010.

[9] CPAC-OtoBox series of automatic controller user manual [Z]. Shenzhen: solid high-tech .2011. 